\title{
STRICTLY NEF DIVISORS
}

\section{FRÉDÉRIC CAMPANA, JUNGKAI A. CHEN AND THOMAS PETERNELL INTRODUCTION}

Given a line bundle $L$ on a projective manifold $X$, the Nakai-Moishezon criterion says that $L$ is ample if and only if

$$
L^{s} \cdot Y>0
$$

for all irreducible subvarieties $Y \subset X$ of dimension $s \leq \operatorname{dim} Y$. Examples show that it is not sufficient to assume that $L \cdot C>0$ for all curves; line bundles with this property are called strictly nef. If however $K_{X}$ is strictly nef, then standard conjectures predict that $K_{X}$ is already ample; this is proved in dimension up to 3 (Kawamata, Miyaoka, see e.g. [Ko92]). If $\operatorname{dim} X=3$ and $-K_{X}$ is strictly nef, then Serrano [Se95] showed that $-K_{X}$ is ample, i.e. $X$ is a Fano threefold. This lead him to set up the following

0.1. Conjecture. Let $X$ be a projective manifold of dimension $n$ and $L$ a strictly nef line bundle on $X$. Then $K_{X}+t L$ is ample for any real $t>n+1$.

A different view to this conjecture is as follows. Consider a strictly nef, but possibly non-ample line bundle. We want to reach ampleness by adding $\epsilon K_{X}$ with $\epsilon$ a small positive number. This might sound strange at first sight, especially if $K_{X}$ is negative. The point, however, is that the cone of curves is locally polyhedral in the area where $K_{X}$ is negative, so that roughly speaking $L$ is already ample in that area. Of course, if $K_{X}$ has some positivity, then things tend to get easier. A difficult case is however when $K_{X}=0$ and simply connected; in that situation Conjecture 0.1 says that strict nefness and ampleness are the same.

Serrano established the conjecture in dimension 2. He also verified the conjecture in dimension 3, with some important exceptions:

0.2. Theorem. ([Se95], Theorem 4.4) Let $X$ be a smooth projective threefold and $L$ strictly nef. Then $K_{X}+t L$ is ample for $t>4$ with the following possible exceptions.

- $X$ is Calabi-Yau and $L \cdot c_{2}(X)=0$;

- $X$ is uniruled with irregularity $q(X) \leq 1$, this includes the case that $X$ is rationally connected;

- $X$ is uniruled with irregularity $q(X)=2$ and $\chi\left(\mathcal{O}_{X}\right)=0$.

Moreover he solved the case $L=-K_{X}$ in dimension 3 .

0.3. Theorem.([Se95], Theorem 3.9) Let $X$ be a smooth projective threefold with $-K_{X}$ strictly nef. Then $X$ is Fano.

$\{$ Fano $\}$

In this paper we settle the two last open cases in Theorem 0.2 and establish also results in higher dimensions:

0.4. Theorem. Let $X$ be a projective manifold of dimension $n$ and $L$ a strictly nef line bundle on $X$. Then $K_{X}+t L$ is ample if $t>n+1$ in the following cases.

$\{$ mainthm $\}$ 
1.) $\operatorname{dim} X=3$ unless (possibly) $X$ is Calabi-Yau with $L \cdot c_{2}=0$;

2.) $\kappa(X) \geq n-2$;

Concerning the structure of this paper: in section 1, we recall some known facts mainly due to Serrano. Theorem 0.4.2 will be proved in $\S 2$. In section 3 we will deal with Albanese maps. In sections 4 and 5, we study Fano fibration and birational maps. Combining all results, we are finally able to prove Theorem 0.4.1. We have a further result in higher dimensions in $\S 6$.

The remaining three-dimensional case when $X$ is Calabi-Yau with $L \cdot c_{2}=0$ is a very hard problem in Calabi-Yau theory and definitely requires very different methods.

\section{Contents}

Introduction 1

1. Basic definitions, known results and main problems 2

2. Results in case of non-negative Kodaira dimension 4

3. The Albanese map $\quad 7$

4. Fano fibrations 9

5. Birational maps 12

6. Higher dimensions 16

References 16

\section{BASIC DEFINITIONS, KNOWN RESUlts AND MAIN PROBLEMS}

For technical reasons we have to consider not only strictly nef line bundles, but also a slight generalization of this notion.

1.1. Definition. Let $X$ be a normal projective variety.

1.) A line bundle $L$ over $X$ is strictly nef, if $L \cdot C>0$ for all irreducible curves $C \subset X$.

2.) $L$ is almost strictly nef, if there is a normal projective variety $X^{\prime}$, a surjective birational holomorphic map $f: X \rightarrow X^{\prime}$ and a strictly nef line bundle $L^{\prime}$ on $X^{\prime}$ such that $L=f^{*}\left(L^{\prime}\right)$.

As usual, $\overline{N E}(X)$ will denote be the Mori cone of (classes of) curves on an $n$ dimensional variety $X$. Via intersection, the canonical line bundle defines a linear functional on $\overline{N E}(X)$. Let

$$
K_{X}^{<0}:=\left\{[C] \mid C \cdot K_{X}<0\right\}
$$

analogously $K_{\bar{X}}^{\geq 0}$. Assume now that $X$ is $\mathbb{Q}$-Gorenstein with at most canonical singularities. Then the Cone theorem asserts that $\overline{N E}(X)$ is generated by $K_{\bar{X}}^{\geq 0}$ and the extremal rays $R_{i}$ with $R_{i} \cdot K_{X}<0$. If $X$ is smooth, then the ray $R_{i}$ contains an extremal rational curve $l_{i}$ with $-(n+1) \leq K_{X} \cdot l_{i}$; if $X$ is singular, the bound for a rational curve in $R_{i}$ is more crude, but depends only on $X$ (see e.g. [KMM87], 4-2-1). This implies

\{larget\} 1.2. Proposition. Let $X$ be a normal n-dimensional projective variety, $\mathbb{Q}-$ Gorenstein with at most canonical singularities. If $L$ is a strictly nef line bundle 
on $X$, then $K_{X}+t L$ is strictly nef for $t \gg 0$. If $X$ is smooth, then $K_{X}+t L$ is strictly nef for $t>n+1$.

Serrano's conjecture 0.1 also makes sense in the singular case:

1.3. Conjecture. Let $X$ be a normal projective variety, $\mathbb{Q}$-Gorenstein with at most canonical singularities. Let $i(X)$ be the index, i.e., the smallest number such that $i(X) K_{X}$ is Cartier. Let $L$ be strictly nef on $X$. Then $K_{X}+t L$ is ample for all $t>i(X)(n+1)$.

It is known since a long time that strictly nef divisors need not be ample; even if they are big. See Ramanujam's example in [Ha70], p.57-58. The first example of a strictly nef non-ample line bundle is due to Mumford, see again [Ha70], p.50-56. Here $X$ is a ruled surface over a curve of genus at least 2, coming from a stable rank 2-bundle of degree 0 . In this situation $L=\mathcal{O}_{\mathbb{P}(E)}(1)$ is strictly nef, but $L^{2}=0$. Of course there must be a class $\alpha \in \overline{N E}(X)$ with $L \cdot \alpha=0$, but $\alpha$ is not represented by an effective curve.

The following Lemma is useful in the sequel

1.4. Lemma. Suppose $L$ is semi-ample (i.e., some multiple of $L$ is spanned) and strictly nef. Then $L$ is ample.

Proof. Since $L$ is semi-ample, $m L$ is spanned for some $m \gg 0$. Let $\varphi: X \rightarrow Y$ be the morphism defined by $m L$. If there is a curve $C$ in a fiber, then $C \cdot L=0$, which is a contradiction. Thus $\varphi$ is finite and thus $L$ is ample.

There are three important prominent special cases of the conjecture, namely when $K_{X}=L,-L, \equiv 0$ respectively.

In the first case, the abundance conjecture predicts that $m K_{X}$ is spanned for a suitable large $m$. So $K_{X}$ will be ample and thus Conjecture 0.1 holds. This is known in dimension up to 3 .

In the second case, Serrano's conjecture predicts that $X$ is Fano if $-K_{X}$ is strictly nef. This is verified by Serrano up to dimension 3 (Theorem 0.3).

In the last case, the conjecture predicts that every strictly nef line bundle on every Ricci-flat manifold is ample. This clearly holds if $X$ is an abelian variety, (see e.g. [Se95], Proposition 1.4). However, it is very subtle if $X$ is Calabi-Yau, even in dimension 3.

We now recall some known results which will be useful later. Lemma 1.5 is particularly crucial since it gives severe numerical restrictions.

1.5. Lemma. ([Se95], Lemma 1.3) Let $X$ be an $n$-dimensional connected projective manifold and $L$ a strictly nef line bundle on $X$. If $K_{X}+t L$ is not ample for some real number $t>n+1$, then $K_{X}^{j} \cdot L^{n-j}=0$ for all $j \geq 0$.

An easy consequence is

1.6. Corollary. (1) If $\left(K_{X}+t L\right)^{n} \neq 0$ for some real number $t$, then $K_{X}+t L$ is ample for any $t>n+1$.

(2) If $p K_{X}+q L$ is ample for some $p, q$, then $K_{X}+t L$ is ample for any $t>n+1$.

1.7. Theorem.([Se95], Theorem 2.3) Let $X$ be a irreducible reduced projective Gorenstein surface and $L$ strictly nef on $X$. Then $K_{X}+t L$ is ample for any real number $t>3$. 
An immediate application of the above two results is the following

1.8. Proposition.([Se95], Proposition 3.1) If $\operatorname{dim} X=3$ and $\left|p K_{X}+q L\right|$ contains an effective non-zero divisor for some integers $p, q$, then $K_{X}+t L$ is ample for any $t>4$. 0.3

Combining all these results, Serrano was able to prove Theorem 0.2 and Theorem

1.9. Remark. Perhaps the best justification for the Conjecture 0.1 is that it holds for $L$ if and only if

$$
L^{\perp} \cap K_{X}^{\perp} \cap \overline{N E}(X)=\{0\},
$$

in $N_{1}(X)$, see Proposition 1.10 below. So the conjecture should be viewed as a statement on the cone $\overline{N E}(X)$, at the points where the intersection number with $K$ and $L$ simultaneously vanish.

By $M E(X)$ we will always denote the cone of movable curves. Its closure is the cone dual to the cone of effective divisors; see [BDPP04] for details.

$\{\mathrm{BDPP}\}$ 1.10. Proposition. Let $X$ be a projective manifold of dimension $n$. Let $L$ be strictly nef and suppose there is some non-zero $\alpha \in \overline{N E}(X)$ such that $\left(K_{X}+t L\right) \cdot \alpha=$ 0 for some $t>n+1$. Then

1.) $K_{X} \cdot \alpha=L \cdot \alpha=0$.

2.) $L^{\perp} \cap K_{X}^{\perp} \cap \partial M E(X) \neq\{0\}$.

Proof. (1) Suppose $L \cdot \alpha \neq 0$. Then $L \cdot \alpha>0$ and therefore $K_{X} \cdot \alpha<0$. By the cone theorem we can write

$$
\alpha=\sum_{i=1}^{N} a_{i} C_{i}+R
$$

with $C_{i}$ extremal rational curves and $K_{X} \cdot R \geq 0$. Since $-K_{X} \cdot C_{i} \leq n+1$ by a standard fact of Mori theory (see e.g. [Mo82]) - otherwise $C_{i}$ deforms in a splitting family - and $t L . C_{i} \geq t>n+1$, for all $i$, we have $\left(K_{X}+t L\right) \cdot C_{i}>0$, which gives a contradiction.

(2) If there is no nonzero $\alpha \in \partial M E(X)$ with $\left(K_{X}+t L\right) \cdot \alpha=0$, then by [BDPP04], Theorem $0.2, K_{X}+t L$ is big. But then $K_{X}+t L$ is ample, by Lemma 1.5.

\section{Results in CASE OF NON-NEGATIVE KodAiRA DIMENSION}

If $X$ is of general type, then Conjecture 0.1 easily holds:

2.1. Proposition. Let $X$ be a projective $n$-dimensional manifold with $\kappa(X)=n$. Let $L$ be strictly nef on $X$. Then $K_{X}+t L$ is ample for any $t>n+1$.

Proof. Let $t>n+1$ be a rational number. By Proposition 1.2, $K_{X}+t L$ is strictly nef. Then $2\left(K_{X}+t L\right)-K_{X}=K_{X}+2 t L$ is nef. Since this holds for any $t>n+1$, we can find a small positive rational number $\epsilon$ such that $(1-\epsilon) K_{X}+2 t L$ is still nef. Since $K_{X}$ is big and since the sum of a nef and a big divisor is again big, $2\left(K_{X}+t L\right)=K_{X}+2 t L$ is big. By the base point free theorem, $K_{X}+t L$ is therefore semi-ample. Since it is strictly nef, it is ample by Lemma 1.4.

If $X$ is not of general type, things are more complicated. Here we want to use the Iitaka fibration. For technical reasons we slightly generalize Conjecture 0.1: 
2.2. Conjecture. $\left(\mathbf{C}_{\mathbf{d}}\right)$ : Let $F$ be a d-dimensional projective manifold. Let $L$ be almost strictly nef. Then, $K_{F}+t L$ is big for any $t>d+1$.

Conjecture $C_{1}$ being obvious, we are now going to prove $C_{2}$ for surfaces with $\kappa=0$.

2.3. Proposition. Let $X$ be a smooth projective surface with $\kappa(X)=0$ and $L$ almost strictly nef. Then $K_{X}+t L$ is big for any $t>3$.

Proof. Fix a rational number $t>3$ and suppose that $K_{X}+t L$ is not big.

By blowing down the $(-1)$-curves $E_{i}$ with $L \cdot E_{i}=0$, we may assume that $K_{X}+$ $t L$ is nef. In fact, if $K_{X}+t L$ is not nef, then there exists a curve $C$ such that $\left(K_{X}+t L\right) \cdot C<0$. Then we also find by the cone theorem a $(-1)$-curve $C$ with $\left(K_{X}+t L\right) \cdot C<0$. Since $K_{X} \cdot C=-1$, we must have $L \cdot C=0$. Thus by a finite sequence of blow-downs we arrive at the situation where $K_{X}+t L$ is nef.

Thus we may now assume that $K_{X}+t L$ is nef; in fact, notice that if $\sigma: X \rightarrow X^{\prime}$ is a sequence of blow-downs of $(-1)$-curves and if $K_{X^{\prime}}+t L^{\prime}$ is big (with $L=\sigma^{*}\left(L^{\prime}\right)$, then $K_{X}+t L$ is big as well.

So if $K_{X}+t L$ is not big, we must have $\left(K_{X}+t L\right)^{2}=0$.

Suppose first $L^{2}>0$. Then from $\left(K_{X}+t L\right)^{2}=0$, from the nefness of $K_{X}+t L$, and from

$$
0=\left(K_{X}+t L\right)^{2}=K_{X} \cdot\left(K_{X}+t L\right)+t L \cdot\left(K_{X}+t L\right),
$$

we obtain $L \cdot\left(K_{X}+t L\right)=0$. Now the Hodge Index Theorem yields $K_{X}+t L \equiv 0$ which is impossible since $\kappa(X)=0$ and $L$ is almost strictly nef.

So suppose $L^{2}=0$. Hence

$$
K_{X}^{2}+2 t\left(K_{X} \cdot L\right)=0
$$

This holds also for all rational numbers $3<t_{0}<t$, because otherwise $K_{X}+t_{0} L$ would be big and then also $K_{X}+t L$ is big. Thus

$$
K_{X}^{2}=0=K_{X} \cdot L \text {. }
$$

The surface $X$ must then be minimal. By taking a finite étale cover, we can assume $X$ to be either an abelian, or a $K 3$-surface.

On an abelian surface however every almost strictly nef line bundle is strictly nef, hence ample by Theorem 1.7. Furthermore, Riemann-Roch shows that a nef line bundle on a $K 3$-surface is either (non-zero) effective or trivial. An effective almost strictly nef line bundle on a $K 3$-surface is immediately seen to be big.

2.4. Remark. Conjecture $\left(C_{2}\right)$ trivially holds also on surfaces of general type and is easily checked in case $\kappa=1$. It should also hold in case $\kappa=-\infty$ but we don't need this.

We need the following technical Lemma. This is presumably well-known to experts. However, we include it here for lack of appropriate reference.

2.5. Lemma. Let $g: X \rightarrow Y$ be a surjective holomorphic map of projective varieties. Let $A, N$ be $\mathbb{Q}$-divisors, with $A$ big on $Y, N$ pseudoeffective (e.g.nef) on $X$. Suppose that $N$ is $g$-big, i.e. big on the general fiber. Then $N+g^{*}(A)$ is big.

Proof. By Kodaira's lemma a big divisor decomposes as $\mathbb{Q}$-divisor into an ample and an effective part. Therefore we assume $A$ to be ample on $Y$. We first claim that we can pick $k \gg 0$ such that $N+g^{*}(k A)$ is big. 
This is a standard fact, seen as follows, by a relative version of Kodaira's Lemma: let $H$ be $g$-ample on $X$. Then choose $m$ such that

$$
g_{*}(m N-H)
$$

has positive rank. This is obviously possible, by the coherence of direct image sheaves, since $N$ is $g$-big, (see e.g. [KMM87,0-3-4]). Now choose $k$ large enough, such that $g_{*}(m N-H)+k A$ has a section. Thus

$$
E:=m N-H+g^{*}(k A)
$$

is effective, and $m N+g^{*}(k A)=H+E$ is the sum of an ample and an effective line bundle, hence big. Thus also $N+g^{*}(k A)$ is big for large $k$, proving our first claim.

We now write $N+g^{*}(k A)$ as the sum of an ample and an effective $\mathbb{Q}$-divisor:

$$
N+g^{*}(k A) \equiv H^{\prime}+E^{\prime} .
$$

Thus

$$
k\left(N+g^{*} A\right) \equiv\left(H^{\prime}+(k-1) N\right)+E^{\prime}
$$

is again the sum of a big and an pseudo-effective divisor. Since a pseudo-effective divisor is by definition in the closure of the effective cone, this sum is again big. Hence $N+g^{*} A$ is big.

\{Iitaka\} 2.6. Theorem. Let $X$ be an n-dimensional connected projective manifold with $\kappa(X)=k \geq 0$. Let $L$ be a strictly nef line bundle on $X$. Suppose that $\left(C_{d}\right)$ holds for $d=n-k$. Then $K_{X}+t L$ is ample for $t>n+1$.

Proof. Let $f: X \rightarrow Y$ be the Iitaka fibration; we may assume $\operatorname{dim} Y=k \geq 1$, because otherwise there is nothing to prove. Let $\pi: \hat{X} \rightarrow X$ be a sequence of blow-ups such that the induced map $\hat{f}: \hat{X} \rightarrow Y$ is holomorphic and such that we can write

$$
\pi^{*}\left(m K_{X}\right)=\hat{f}^{*}(A)+E
$$

with a suitable large $m$, an ample divisor $A$ on $Y$ and an effective divisor $E$. We also have an equality $K_{\hat{X}}=\pi^{*}\left(K_{X}\right)+E^{\prime}$ for some effective $E^{\prime}$. Let us furthermore set $\hat{L}=\pi^{*}(L)$.

By $\left(C_{d}\right)$ applied to the general fiber $\hat{F}$ of $\hat{f}$, the divisor $K_{\hat{F}}+t \hat{L}$ is big for $t>d+1$. Thus $K_{\hat{X}}+t \hat{L}$ is $\hat{f}$-big for $t>d+1$. Now Lemma 2.5 therefore applies, with $N=\pi^{*}\left(K_{X}\right)+t \hat{L}$ and with $D=E^{\prime}$ and shows the bigness of $K_{\hat{X}}+t \hat{L}+\hat{f}^{*}(A)$ for $t \gg 0$. Hence can write

$$
K_{\hat{X}}+t \hat{L}+\hat{f}^{*}(A) \equiv H+\Delta
$$

for some ample $H$ and some effective $\Delta$. Thus by $\left(^{*}\right)$, we have

$(m+1)\left(K_{\hat{X}}+t \hat{L}\right)=K_{\hat{X}}+t \hat{L}+\pi^{*} m K_{X}+m E^{\prime}+m t \hat{L} \equiv(H+m t \hat{L})+\left(\Delta+E+m E^{\prime}\right)$, which is big.

Therefore $K_{\hat{X}}+t \hat{L}$ is big. It follows that $K_{X}+t L=\pi_{*}\left(K_{\hat{X}}+t \hat{L}\right)$ is big for $t \gg 0$. Moreover, $K_{X}+t L$ is strictly nef for $t \gg 0$ by Proposition 1.2. Hence $\left(K_{X}+t L\right)^{n}>0$ for $t$ large. By Corollary 1.6, we conclude.

Combining (2.3) and (2.6) we obtain:

\{Iitaka2\} 2.7. Corollary. Let $X$ be an n-dimensional connected projective manifold with $\kappa(X) \geq n-2$. Let $L$ be a strictly nef line bundle on $X$. Then $K_{X}+t L$ is ample for any $t>n+1$. 


\section{The Albanese map}

We now study Conjecture 0.1 on projective manifolds $X$ with positive irregularity $q(X)>0$.

3.1. Theorem. Let $X$ be a smooth projective threefold, L strictly nef. Suppose there exists a non-constant map $g: X \rightarrow A$ to an abelian variety. Then $K_{X}+t L$ is ample for any real number $t>4$.

Proof. Notice that it suffices by Lemma 1.5 and Corollary 1.6 to prove the claim just for one $t>4$. For any integer $s>0$, we let $D_{s}:=2 K_{X}+2 s L$. We claim that

$$
\mathcal{F}_{s}:=g_{*}\left(2 K_{X}+2 s L\right)
$$

satisfies a generic vanishing theorem for $t$ sufficiently large. That is to say that $\mathcal{F}_{s}$ satisfies the equivalent conditions of [Ha04] Theorem 1.2, and thus we have a chain of inclusions

where

$$
V^{0}\left(\mathcal{F}_{s}\right) \supset V^{1}\left(\mathcal{F}_{s}\right) \ldots \supset V^{n}\left(\mathcal{F}_{s}\right)
$$

$$
V^{i}\left(\mathcal{F}_{s}\right):=\left\{P \in \operatorname{Pic}^{0}(A) \mid h^{i}\left(A, \mathcal{F}_{s} \otimes P\right) \neq 0\right\} .
$$

Given this claim for granted for the time being, we proceed as follows. Since $\mathcal{F}_{s}$ is a non-zero sheaf for $s \gg 0$, one concludes that $V^{0}\left(\mathcal{F}_{s}\right) \neq \emptyset$ for large $s$. For otherwise, $V^{i}\left(\mathcal{F}_{s}\right)=\emptyset$ for all $i$, which implies that the Fourier-Mukai transform of $\mathcal{F}_{s}$ is zero. This is absurd e.g. by [Mu81], Theorem 2.2.

Therefore, fixing $s$ sufficiently large, we have

$$
h^{0}\left(X, 2 K_{X}+2 s L+g^{*} P\right) \neq 0
$$

for some $P \in \operatorname{Pic}^{0}(A)$. Of course $P$ depends on $s$. Notice that if $2 K_{X}+2 s L+g^{*} P$ has a section without zeroes, then $-K_{X}$ is strictly nef, hence $X$ is Fano and $q(X)=0$ by Corollary 0.3 , which contradicts our assumption.

Now let $L^{\prime}$ be a $\mathbb{Q}$-divisor such that

$$
2 s L^{\prime}=2 s L+g^{*} P .
$$

Choose a positive integer $m$ such that $2 m s L^{\prime}$ is Cartier. Then apply Proposition 1.8 to $2 m s L^{\prime}$ so that the bundle

$$
K_{X}+t L^{\prime}=K_{X}+\frac{t}{2} L+g^{*} P^{\prime}
$$

is ample for large divisible $t$ and a suitable multiple $P^{\prime}$ of $P$. Hence $K_{X}+t L$ is ample, and the claim of the theorem is proved for one $t>4$ and therefore for all $t>4$.

It remains to prove the generic vanishing claim. First note that $K_{X}+t_{0} L$ is $g$-big for some $t_{0}>3$ (Theorem 1.7). Fix an ample line bundle $H$ on $A$. From Lemma 2.5 we deduce that $a\left(K_{X}+2 s L\right)+g^{*} H$ is nef and big for $a>0$ and $s \geq t_{0}$. We choose $s$ to be an integer $s>4$. Set

$$
D_{0}:=2\left(K_{X}+2 s L\right)+g^{*} H .
$$

Then $D_{0}-K_{X}$ is again nef and big. By the Base Point Free Theorem, $m D_{0}$ is therefore spanned for some $m \gg 0$.

Take $D$ a general smooth member in $\left|m D_{0}\right|$. Then we have

$$
2 K_{X}+2 s L+g^{*} H \equiv K_{X}+\frac{1}{2} g^{*} H+\frac{1}{2 m} D,
$$


where $\left(X, \frac{1}{2 m} D\right)$ is Kawamata log-terminal (klt), see e.g. [KM98], 2.34 or [KMM87], 0.2.10 for definition. By the vanishing theorem of Kollár (cf. [Ko95], Theorem 10.19.2), we have

$$
\left.H^{j}\left(A, g_{*}\left(2 K_{X}+2 s L\right) \otimes H\right)\right)=0, \text { for all } j>0
$$

and moreover

$\left.H^{j}\left(A, \mathcal{F}_{s} \otimes H \otimes P\right)=H^{j}\left(A, g_{*}\left(2 K_{X}+2 s L\right) \otimes H \otimes P\right)\right)=0$ for all $j>0, P \in \operatorname{Pic}^{0}(\mathrm{~A})$.

In other words, per definitionem the sheaf $\mathcal{F}_{s} \otimes H$ is $I T^{0}$ for all ample line bundles $H$.

Next, let $M$ be any ample line bundle on the dual abelian variety $\hat{A}$ and $\phi: \hat{A} \rightarrow$ $A$ is the isogeny defined by $M$. Let $\hat{M}$ be the Fourier-Mukai transform of $M$ on $A$ and let $\hat{M}^{\vee}$ be its dual. By [Mu81] Proposition 3.11,

$$
\phi^{*}\left(\hat{M}^{\vee}\right) \cong \oplus^{h^{0}(M)} M .
$$

Let $\hat{g}: \hat{X}:=X \times_{A} \hat{A} \rightarrow \hat{A}$ be the base change with the induced map $\varphi$ : $\hat{X} \rightarrow X$ being étale. Clearly, $K_{\hat{X}}=\varphi^{*} K_{X}$ and $\varphi^{*} L$ is strictly nef on $\hat{X}$. Let $\mathcal{G}_{s}:=\hat{g}_{*}\left(\varphi^{*}\left(2 K_{X}+2 s L\right)\right)$. By applying the above argument to $\varphi^{*} D_{s}$, we see that $\mathcal{G}_{s} \otimes M$ is $I T^{0}$ for all $M$. Thus

$$
\begin{aligned}
\phi^{*}\left(\mathcal{F}_{s} \otimes \hat{M}^{\vee}\right) & =\phi^{*}\left(g_{*}\left(D_{s} \otimes \hat{M}^{\vee}\right)\right) \\
& =\phi^{*} g_{*}\left(\left(D_{s} \otimes g^{*} \hat{M}^{\vee}\right)\right) \\
& =\hat{g}_{*} \varphi^{*}\left(\left(D_{s} \otimes g^{*} \hat{M}^{\vee}\right)\right) \\
& =\hat{g}_{*}\left(\varphi^{*} D_{s} \otimes \varphi^{*} g^{*} \hat{M}^{\vee}\right) \\
& =\hat{g}_{*}\left(\varphi^{*} D_{s} \otimes \hat{g}^{*} \phi^{*} \hat{M}^{\vee}\right) \\
& =\hat{g}_{*}\left(\varphi^{*} D_{s} \otimes \hat{g}^{*}(\oplus M)\right) \\
& =\oplus\left(\hat{g}_{*} \varphi^{*} D_{s} \otimes M\right)
\end{aligned}
$$

which is $I T^{0}$.

Since $\mathcal{O}_{A}$ is a direct summand of $\phi_{*} \phi^{*} \mathcal{O}_{A}$, it follows that $\mathcal{F}_{s} \otimes \hat{M}^{\vee}$ is $I T^{0}$. Now our claim follows from [Ha04], Theorem 1.2.

We introduce the following notation:

$$
\tilde{q}(X):=\max \{q(\tilde{X}) \mid \tilde{X} \rightarrow X \text { is finite étale }\} .
$$

\{girreg3\} 3.2. Corollary. Let $X$ be a smooth projective threefold, L strictly nef. Suppose that $\tilde{q}(X)>0$. Then $K_{X}+t L$ is ample for $t>4$.

Proof. By the previous theorem we only have to treat the case that $q(X)=0$. Then we choose a finite étale cover $h: \tilde{X} \rightarrow X$ such that $q(\tilde{X})>0$. Hence $K_{\tilde{X}}+h^{*}(L)$ is ample for $t>n+1$ and so does $K_{X}+t L$.

3.3. Remark. There are two obstacles for extending Theorem 3.1 to all dimensions. The first is the use of Proposition 1.8 which has to be extended to higher dimensions, at least in our situation. Serrano's proof requires that the Conjecture 0.1 holds for Gorenstein singular subvarieties of codimension 1 . Thus we will consider Gorenstein varieties in Proposition 3.4, constructing a section in $2 K_{\hat{X}}+2 t \hat{L}+\hat{P}$ with $\hat{P}$ numerically trivial, where $\pi: \hat{X} \rightarrow X$ is a desingularization and $\hat{L}=\pi^{*}(L)$. Thus we are forced to work with almost strictly nef line bundles.

The second obstacle is the $g$-bigness of $K_{X}+t L$. This means that $K_{F}+t L_{F}$ is big 
for the general fiber $F$ of $g$. Thus it is natural to argue by induction on the dimension, but of course we are far from proving the conjecture for arbitrary manifolds with vanishing irregularity.

3.4. Proposition. Let $X$ be an irreducible reduced projective Gorenstein variety with desingularization $\pi: \hat{X} \rightarrow X$ and let $\hat{L}=\pi^{*}(L)$. Let $g: X \rightarrow A$ be a nonconstant map to an abelian variety and let $L$ be a strictly nef line bundle on $X$. Suppose that $K_{\hat{X}}+t_{0} \hat{L}$ is $(g \circ \pi)$-big for some $t_{0}$. Then

1.) The sheaf $\hat{\mathcal{F}}=g_{*} \pi_{*}\left(2 K_{\hat{X}}+2 t \hat{L}\right)$ satisfies the generic vanishing theorem

$$
V^{0}(\hat{\mathcal{F}}) \supset V^{1}(\hat{\mathcal{F}}) \ldots \supset V^{n}(\hat{\mathcal{F}})
$$

for $t \gg 0$.

2.) If $t \gg 0$, then there exists $\hat{P} \in \operatorname{Pic}^{0}(\hat{X})$ such that

$$
H^{0}\left(2 K_{\hat{X}}+2 t \hat{L}+\hat{P}\right) \neq 0 \text {. }
$$

Proof. (1) This is just what the second part of the proof of Theorem 3.1 - applied to $g \circ \pi$ - gives.

(2) By (1) and the first arguments in the proof of Theorem 3.1, we obtain

$$
H^{0}\left(2 K_{\hat{X}}+2 \hat{L}+\hat{P}\right) \neq 0
$$

for some $\hat{P} \in \operatorname{Pic}^{0}(\hat{X})$.

\section{FANO FibRATIONS}

We shall now complete the proof of Theorem 0.4.1. Observe that due to Theorem 0.2 and and Theorem 3.1, the only cases left are uniruled threefolds with $q=0$. These cases are thus settled by $4.1 / 4.2$ and $5.1 / 5.2$ below.

In this section we settle the cases of del Pezzo fibrations over curves and conic bundles with relative Picard number 1 over surfaces.

4.1. Proposition. Let $X$ be a smooth projective threefold, L strictly nef on $X$. Suppose that $X$ carries an extremal contraction $f: X \rightarrow B$ to a curve $B$. Then $K_{X}+t L$ is ample for large $t$.

Proof. If $-K_{X}$ and $L$ are proportional, then $X$ is Fano by Serrano's theorem, see 0.3 . Thus we may assume that $-K_{X}$ and $L$ are not proportional. Since $f$ is an extremal contraction, we have $\rho(X)=\rho(B)+1$, hence $\rho(X)=2$, so one can arrange that $\overline{N E}(X) \subset\left(p K_{X}+q L\right)^{>0}$ for some suitable $p, q$. By Kleiman's criterion, $p K_{X}+q L$ is ample and hence by Corollary 1.6, we are done.

4.2. Proposition. Let $X$ be a threefold which carries a conic bundle $f: X \rightarrow S$ with $\rho(X / S)=1$. If $L$ is strictly nef on $X$, then $K_{X}+t L$ is ample for $t>4$.

Proof. By Corollary 3.2 we may assume that $q(S)=0$, even after a finite étale cover of the smooth surface $S$.

Suppose that $K_{X}+t L$ fails to be ample. Then by Lemma 1.5, we have $K_{X}^{j}$. $L^{3-j}=0$ for all $j \geq 0$.

Since $\rho(X / S)=1$, we find a positive number $t_{0}$ such that

$$
K_{X}+t_{0} L=f^{*}\left(K_{S}+M\right)
$$


with a $\mathbb{Q}$-divisor $M$ on $S$. We cube the equation $t_{0} L=-K_{X}+f^{*}\left(K_{S}+M\right)$ to obtain

$0=3 K_{X}^{2} \cdot f^{*}\left(K_{S}+M\right)-3 K_{X} \cdot f^{*}\left(K_{S}+M\right)^{2}=3 K_{X}^{2} \cdot f^{*}\left(K_{S}+M\right)+6\left(K_{S}+M\right)^{2}$.

¿From $K_{X} \cdot t_{0} L^{2}=0$ we get $K_{X}^{2} \cdot f^{*}\left(K_{S}+M\right)=0$, hence in total

$$
\left(K_{S}+M\right)^{2}=0 .
$$

By applying Proposition 1.10 to $L$, we find $\alpha \in \overline{M E}(X)$ such that

$$
K_{X} \cdot \alpha=L \cdot \alpha=0,
$$

in particular $D \cdot \alpha=0$. Introducing $\gamma=\phi_{*}(\alpha) \in \overline{M E}(S)$, we obtain

$$
\left(K_{S}+M\right) \cdot \gamma=0 .
$$

Notice that $\overline{M E}(S)$ is nothing than the nef cone, so $\gamma$ is a nef class. Next notice that we may choose $\gamma$ rational. In fact, since the rational points are dense in the nef cone of $S$ and since neither $K_{S}+M$ nor $-\left(K_{S}+M\right)$ are strictly positive functionals on the nef cone, we find rational points $x$ and $y$ in the nef cone such that

$$
\left(K_{S}+M\right) \cdot x \geq 0 ;\left(K_{S}+M\right) \cdot y \leq 0 .
$$

We may assume strict inequality in both cases, otherwise we are already done, setting $x=\gamma$, resp. $y=\gamma$. Then choose $\lambda>0$ such that

$$
\left(K_{S}+M\right) \cdot(x+\lambda y)=0 .
$$

Noticing that $\lambda \in \mathbb{Q}$, we may substitute $\gamma$ by $x+\lambda y$, so that we may now assume $\gamma$ to be rational.

Now multiply $\gamma$ suitably to obtain a nef line bundle $G$ such that

$$
\left(K_{S}+M\right) \cdot G=0 .
$$

If now $G^{2}>0$, then the Hodge Index theorem gives $K_{S}+M=0$, so that $H^{0}\left(m\left(K_{X}+t_{0} L\right)\right) \neq 0$ for positive integers $m$ such that $m t_{0} \in \mathbb{N}$. By Proposition 1.8, $K_{X}+t L$ is ample for $t>4$.

Thus we may assume that $G^{2}=0$. Together with $\left(K_{S}+M\right)^{2}=\left(K_{S}+M\right) \cdot G=0$, one has $\left(K_{S}+M+\tau G\right)^{2}=0$ for all $\tau$.

Let $C \subset S$ be an irreducible curve. Then

$$
\begin{gathered}
t_{0}^{2} L^{2} \cdot f^{*}(C)=\left(f^{*}\left(K_{S}+M\right)-K_{X}\right)^{2} \cdot f^{*}(C)=-2 f^{*}\left(K_{S}+M\right) \cdot K_{X} \cdot C+K_{X}^{2} \cdot f^{*}(C)= \\
=4\left(K_{S}+M\right) \cdot C-\left(4 K_{S}+\Delta\right) \cdot C=(M-\Delta) \cdot C .
\end{gathered}
$$

The last equation is explained as follows. $\Delta$ denoting the discriminant locus, it is well-known ( see e.g. [My83]) that

$$
f_{*}\left(K_{X}^{2}\right)=-\left(4 K_{S}+\Delta\right) .
$$

Now we restrict ourselves to curves $C$ with $C^{2} \geq 0$. Then clearly $L^{2} \cdot f^{*}(C) \geq 0$, hence

in particular

$$
(M-\Delta) \cdot C \geq 0,
$$

$$
(M-\Delta) \cdot G \geq 0 .
$$

Moreover we have a strict inequality in (2) unless $C_{0}=\emptyset$ and $L^{2} \cdot f^{*}(C)=0$. The inequality (3) says in particular that $M$ is pseudo-effective. Thus the equation $\left(K_{S}+M\right) \cdot G=0$ forces $\kappa(S) \leq 1$. 
(I) We first assume $\kappa(S)=-\infty$. Then $S$ is a rational surface, since we may assume $\tilde{q}(S)=0$. The case that $S=\mathbb{P}^{2}$ is easy and left to the reader. So we may assume that $\pi: S \rightarrow S_{0}$ is a succession of blow-ups, where $S_{0}$ is a ruled surface with minimal section $C_{0}$ that $C_{0}^{2}=-e$.

Now we write

$$
\begin{gathered}
K_{S}+M=\pi^{*}\left(\alpha_{1} C_{0}+\beta_{1} F\right)+E_{1} \\
G=\pi^{*}\left(\alpha_{2} C_{0}+\beta_{2} F\right)+E_{2},
\end{gathered}
$$

where $E_{1}, E_{2}$ are divisors supported on exceptional curves.

If $\alpha_{2}=0$, then it is clear that $E_{2}=0$ and $G=\beta_{2} \pi^{*} F$. Then $\left(K_{S}+M\right) \cdot G=0$ gives $\alpha_{1}=0$ and $\left(K_{S}+M\right)^{2}=0$ gives $E_{1}=0$. So $K_{S}+M=\beta_{1} \pi^{*} F$, and we are done, since $K_{S}+M$ is effective and therefore $K_{X}+t_{0} L$ is effective.

If $\alpha_{2} \neq 0$, take $\tau=\frac{-\alpha_{1}}{\alpha_{2}}$, then

$$
K_{S}+M+\tau G=\left(\beta_{1}+\tau \beta_{2}\right) \pi^{*} F+E_{1}+\tau E_{2} .
$$

$\left(K_{S}+M+\tau G\right)^{2}=0$ gives $\left(E_{1}+\tau E_{2}\right)^{2}=0$. It implies $E_{1}+\tau E_{2}=0$ by the negativity of intersection form of exceptional divisors.

Let $\delta:=\beta_{1}+\tau \beta_{2}$. If $\delta \neq 0$, then $K_{S}+M=-\tau G+\delta \pi^{*} F$. Again, one has $\alpha_{2}=G \cdot \pi^{*} F=0$ which is absurd.

Therefore

$$
K_{S}+M=-\tau G .
$$

By (3) we have $M \cdot G \geq 0$, therefore $K_{S} \cdot G \leq 0$. By Riemann-Roch and the obvious vanishing $H^{0}\left(K_{S}-G\right)=0$, we have

$$
h^{0}(S, G) \geq \chi\left(\mathcal{O}_{S}\right)=1 .
$$

Hence $G$ is effective. $G$ is non-zero for otherwise $K_{X}+t_{0} L \equiv 0$, hence $-K_{X}$ is strictly nef and thus $X$ is Fano. Therefore $m\left(K_{X}+t_{0} L\right)$ is effective for some $m \in \mathbb{Z}$ and we are done in Case (I) by Proposition 1.8 again.

(II) Now suppose that $\kappa(S) \geq 0$. Let

$$
\sigma: S \rightarrow S_{0}
$$

be the minimal model. Since $\kappa(S) \geq 0$, we conclude by (2) that

$$
K_{S} \cdot G=M \cdot G=0 .
$$

Hence

$$
0=\sigma^{*}\left(K_{S_{0}}\right) \cdot G+\sum a_{i} A_{i} \cdot G
$$

with $A_{i}$ the $\sigma$-exceptional curves and $a_{i}$ suitable positive rational numbers. Thus $G=\sigma^{*}\left(G_{0}\right)$ with a nef line bundle $G_{0}$ on $S_{0}$; observe that $K_{S_{0}} \cdot G_{0}=0$ and that $G_{0}^{2}=0$.

Suppose that $\kappa(S)=1$. Then we consider the Iitaka fibration $g: S_{0} \rightarrow B$ to the curve $B$. By Theorem 3.1, we may assume that $B=\mathbb{P}_{1}$. We conclude that $G_{0}$ is a sum of fibers of $g$. Thus $G$ is a sum of fibers of $g \circ \sigma$. Now consider the composed map $h: X \rightarrow B$. Then it follows that $h_{*}(\alpha)$ consists of finitely many points. This means that we can find a fiber of $h$ such that $K_{X}+t L \mid F$ is not ample for large $t$. Thus $K_{F}+t L_{F}$ is not ample. If (the reduction of) $F$ is irreducible, this contradicts Theorem 1.7. If $F_{i}$ is a component of $F$ with multiplicity $a_{i}$, then $a_{i} K_{F_{i}}+t L_{F_{i}}$ is a subsheaf of $K_{F}+t L_{F} \mid L_{F_{i}}$, and the contradiction is the same. 
Finally we have to treat the case $\kappa(S)=0$. Here we may assume that $S_{0}$ is $\mathrm{K} 3$ by Corollary 3.2. If $G_{0}^{2}=0$, then by Riemann-Roch $\kappa\left(G_{0}\right)=1$. Hence some multiple of $G_{0}$ is spanned, defining a morphism $g: S \rightarrow B$. Since the divisor $M_{0}$ must be supported on fibers of $g$, so does $\Delta$. Thus we conclude by (3) for $b \in B$ that

$$
L^{2} \cdot X_{b}=0 \text {. }
$$

But for general $b$, the fiber $S_{0, b}$ is an elliptic curve and $X_{b}$ is a $\mathbb{P}_{1}$-bundle over $S_{0, b}$ since $\Delta$ does not meet $S_{0, b}$. Moreover $L \mid X_{b}$ is strictly nef, hence ample, contradicting $L^{2} \cdot X_{b}=0$.

4.3. Remark. Suppose in (4.2) that $\phi: X \rightarrow S$ is a conic bundle, but not necessarily with $\rho(X / S)=1$. Then all arguments still remain valid if $K_{X}+t_{0} L$ is the $\phi$-pull-back of a $\mathbb{Q}$-bundle on $S$, for some rational $t_{0}$.

\section{BirationAL MAPS}

In order to prove Conjecture 0.1 in the remaining uniruled cases, it is natural to consider the Mori program. If $X$ admits a contraction contracting a divisor to a point, the situation is easily understood.

5.1. Theorem. Let $X$ be a smooth projective threefold, L strictly nef on $X$. Suppose that $X$ admits a birational Mori contraction $\phi: X \rightarrow Y$ contracting the exceptional divisor $E$ to a point. Then $K_{X}+t L$ is ample for $t>n+1$.

Proof. Suppose that $K_{X}+t L$ is not ample. Write

$$
K_{X}=\phi^{*}\left(K_{Y}\right)+a E ;
$$

then $a \in\left\{2,1, \frac{1}{2}\right\}$ ([Mo82]). Possibly after replacing $L$ by $2 L$ in case $a=\frac{1}{2}$, we can moreover write

$$
L=\phi^{*}\left(L^{\prime}\right)-b E
$$

with a line bundle $L^{\prime}$ on $Y$. Notice that $b>0$ since $L$ is strictly nef. Introduce

$$
D=b K_{X}+a L ; D^{\prime}=b K_{Y}+a L^{\prime} .
$$

Since $L^{\prime}$ is again strictly nef, $K_{Y}+t L^{\prime}$ is strictly nef for $t \gg 0$. Using Lemma 1.5 on $X$ it is a simple matter to verify

$$
\left(K_{Y}+t L^{\prime}\right)^{3}>0
$$

for large $t$, so that $K_{Y}+t L^{\prime}$ is ample by Lemma 1.5. Hence we find positive integers $p, q$ such that $p K_{Y}+q L^{\prime}$ is spanned. Choose $S \in\left|p K_{Y}+q L^{\prime}\right|$ smooth. Now a simple calculation shows that

$$
D^{\prime 2} \cdot\left(p K_{Y}+q L^{\prime}\right)=D^{\prime} \cdot\left(p K_{Y}+q L^{\prime}\right)^{2}=0 .
$$

Thus $D_{S}^{\prime} \cdot\left(p K_{Y}+t L^{\prime}\right)_{S}=0$. Moreover $\left(D_{S}^{\prime}\right)^{2}=0$. Hence $D_{S}^{\prime} \equiv 0$ by the Hodge index theorem. Consequently $D^{\prime} \equiv 0$ and therefore $D \equiv 0$ so that $a L \equiv-b K_{X}$. Therefore $X$ is Fano by Corollary 0.3 and $K_{X}+t L$ is ample for $t>4$, contradiction.

In case that the contraction $\phi: X \rightarrow Y$ contracts a divisor to a curve $C$, the situation is more involved. The reason is that the induced line bundle $L^{\prime}$ on $Y$ is not necessarily strictly nef, in fact we can have $L^{\prime} \cdot C \leq 0$. We have already shown that if $X$ admits a Mori fibration or a divisorial contraction to a point, then the conjecture holds. Since $X$ is smooth, it remains to consider the case that all the extremal rays produce a divisorial contraction to a nonsingular curve. 
5.2. Proposition. Let $X$ be a smooth uniruled threefold, L strictly nef on $X$. Suppose that all extremal contractions on $X$ contract a divisor to a curve. Then $K_{X}+t L$ is ample for large $t$.

Proof. (a) Let us fix some notations first. Let $\phi_{i}, i \in I \subset \mathbb{N}$ be the extremal contractions on $X$, with exceptional divisor $E_{i}$. Let $C_{i}:=\phi_{i}\left(E_{i}\right)$ so that $E_{i}$ is a $\mathbb{P}_{1}$ bundle over $C_{i}$. Let $\left[l_{i}\right] \in K_{X}^{<0}$ denote the class of the contracted ruling lines in $E_{i}$, so that $-K_{X} \cdot l_{i}=1$. Let

$$
\mu:=\min \left\{\frac{L \cdot l_{i}}{-K_{X} \cdot l_{i}} \mid i \in I\right\}=\min \left\{L \cdot l_{i} \mid i \in I\right\} \in \mathbb{N} .
$$

Reorder $I$ so that $\phi_{1}, \ldots, \phi_{n}$ are exactly those contractions with

$$
L \cdot l_{i}=\mu \text {. }
$$

Then the divisor

$$
D:=L+\mu K_{X}
$$

is nef, as a consequence of the cone theorem and the definition of $\mu$. Moreover, if $D \cdot B=0$ for some $B \in \overline{N E}(X)$, then $K_{X} \cdot B \leq 0$. In other words,

$$
D^{\perp} \cap \overline{N E}(X) \subset K_{\bar{X}}^{\leq 0} \text {. }
$$

In particular, if $B$ is an effective curve, then $D \cdot B=0$ forces $K_{X} \cdot B<0$, because otherwise $K_{X} \cdot B=0$, hence $L \cdot B=0$, contradicting the strict nefness of $L$.

Our goal is to show that some multiple $m D=m L+m \mu K_{X}$ is effective, so that we are done by Proposition 1.8 .

Let $\phi=\phi_{1}: X \rightarrow X_{1}=X^{\prime}$ be the contraction of $E=E_{1}$. Let $[l]=\left[l_{1}\right]$ and set $L^{\prime}:=\left(\phi_{*} L\right)^{* *}$, the double dual of $\phi_{*} L$. Furthermore let

$$
D^{\prime}:=L^{\prime}+\mu K_{X^{\prime}}, D:=\phi^{*}\left(D^{\prime}\right)=L+\mu K_{X} .
$$

Let $C=\phi(E)$ and $C_{0}$ be the minimal section in $E$ with $C_{0}^{2}=-e$, in the notation of $[\mathrm{Ha} 77, \mathrm{~V} .2]$.

(b) We introduce the following numbers

$$
\tau:=L^{\prime} \cdot C, \sigma:=K_{X^{\prime}} \cdot C, \gamma=c_{1}\left(N_{C / X}^{*}\right) .
$$

Furthermore, let $g$ be the genus of $C$ and $\chi=2-2 g$.

(b.1) First we treat the case $L^{\prime} \cdot C>0$ so that $L^{\prime}$ is strictly nef. Then by induction on $\rho$, the bundle $K_{X^{\prime}}+t L^{\prime}$ is ample, for $t>4$. Let $t_{0}=\frac{1}{\mu}$. Then $K_{X^{\prime}}+t_{0} L^{\prime}$ is nef, since $D^{\prime}$ is nef. Let $\epsilon>0$ be a small positive number. Then

$$
K_{X^{\prime}}+\frac{t_{0}}{1-\epsilon} L^{\prime}
$$

is big, otherwise we would have $\left(K_{X^{\prime}}+t L^{\prime}\right)^{3}=0$ for all $t$ which is absurd. Now the base point free theorem implies that some multiple $m\left(K_{X^{\prime}}+t_{0} L^{\prime}\right)$ is spanned, hence $m^{\prime} D^{\prime}$ is spanned, so does $L+\mu K_{X}$ and we are done by Lemma 1.4.

Thus we are reduced to

$$
L^{\prime} \cdot C \leq 0
$$

Hence $K_{X^{\prime}} \cdot C \geq 0$, and $C$ is rigid, since $L^{\prime} \cdot C^{\prime}>0$ for every irreducible effective curve $C^{\prime} \neq C$ on $X^{\prime}$. 
(b.2) We claim that:

$$
D^{\prime} \cdot C>0 .
$$

In fact, we need only to exclude the case: $D^{\prime} \cdot C=0$. Assuming $D^{\prime} \cdot C=0$, we obtain

$$
L^{\prime} \cdot C+\mu K_{X^{\prime}} \cdot C=0
$$

and

$$
L_{E} \equiv-\mu K_{X} \mid E .
$$

Since $L \cdot C_{0}>0$, we have $K_{X^{\prime}} \cdot C_{0}<0$, hence $C_{0}$ moves. Since $C$ is rigid, $C_{0}$ can move only inside $E$, hence $e \leq 0$. Write $N_{E}^{*} \equiv C_{0}+\lambda l$. Then it is easily checked that $\lambda=\frac{1}{2} \gamma+\frac{1}{2} e$ in the notations of [Ha 77]; so that

$$
N_{E}^{*}=C_{0}+\left(\frac{1}{2} \gamma+\frac{1}{2} e\right) l .
$$

Since $L_{E}$ is strictly nef, so is $-K_{X} \mid E-N_{E}^{*}=C_{0}+(e+2-2 g-\lambda) l$, so that we conclude:

hence

$$
e+2-2 g-\frac{1}{2} \gamma-\frac{1}{2} e \geq \frac{e}{2},
$$

$$
2-2 g \geq \frac{1}{2} \gamma,
$$

with strict inequality for $e=0$, since on those ruled surfaces all strictly nef line bundles are ample.

By the adjunction formula we have $\gamma=\sigma+(2-2 g)$, hence $\sigma \leq 2-2 g$. Since $\sigma \geq 0$, we obtain $g \leq 1$. But a strictly nef divisor on a ruled surface over a rational or an elliptic curve is ample, hence the inequality $\left({ }^{* *}\right)$ is strict. Thus $g=e=0$ and $\sigma<2, \gamma \leq 3$. So

$$
N_{C}^{*}=\mathcal{O}(k) \oplus \mathcal{O}(k)
$$

with $0<\gamma=2 k \leq 3$, hence $k=1$ and $\sigma=0$. So $K_{X^{\prime}} \cdot C=0=L^{\prime} \cdot C$, and $L^{\prime}$ is nef. If for large $t$, the nef bundle $K_{X^{\prime}}+t L^{\prime}$ is big, then we conclude as in the case $L^{\prime} \cdot C>0$. So we may assume that $\left(K_{X^{\prime}}+t L^{\prime}\right)^{3}=0$ for all $t$. Then $K_{X^{\prime}}^{3}=0$. However $K_{X}^{3}=0$ forces $K_{X^{\prime}}^{3}=-2$, contradiction. Thus we must have

$$
D^{\prime} \cdot C>0 .
$$

(c) Case: $D^{\prime \perp} \cap \overline{N E}(X) \subset K_{X^{\prime}}^{\perp}$.

We are going to rule out this case. Assume first that there is an irreducible curve $B^{\prime} \in \overline{N E}\left(X^{\prime}\right)$ such that $D^{\prime} \cdot B^{\prime}=0$. Necessarily $B^{\prime} \neq C$. By assumption, $K_{X^{\prime}} \cdot B^{\prime}=$ 0 . Let $B$ be the strict transform of $B^{\prime}$ in $X$. Then $D \cdot B=0$. Since $E \cdot B \geq 0$, we also get $K_{X} \cdot B \geq 0$. Since $L \cdot B>0$ and $D \cdot B=0$, this is impossible.

Hence $D^{\prime}$ is strictly nef and by induction, $K_{X^{\prime}}+t L^{\prime}$ is ample for large $t$. On the other hand, we may assume that $D^{\prime}$ is not ample, otherwise we are done by Corollary 1.6. Hence there exists a nonzero class $B^{*} \in \overline{N E}\left(X^{\prime}\right)$ with $D^{\prime} \cdot B^{*}=0$, hence $K_{X^{\prime}} \cdot B^{*}=0$, by our assumption (c). This is absurd, since then $L^{\prime} \cdot B^{*}=0$; on the other hand $K_{X^{\prime}}+t L^{\prime}$ is ample.

(d) Case: $D^{\prime} \cap \overline{N E}(X) \not \subset K_{X^{\prime}}^{\perp}$.

Then we find $B^{\prime} \in \overline{N E}\left(X^{\prime}\right)$ such that $D^{\prime} \cdot B^{\prime}=0$ and $K_{X^{\prime}} \cdot B^{\prime}<0$. Since $D^{\prime}$ is nef, we also find an extremal curve $l^{\prime}$ with $D^{\prime} \cdot l^{\prime}=0$. Let $\phi^{\prime}: X^{\prime} \rightarrow X^{\prime \prime}$ be the associated contraction. 
(d.1) Suppose that $\operatorname{dim} X^{\prime \prime} \leq 2$. Observe that $D^{\prime}=\phi^{\prime *}\left(D^{\prime \prime}\right)$ with a nef bundle $D^{\prime}$ on $X^{\prime \prime}$. So if $\operatorname{dim} X^{\prime \prime} \leq 1$, the bundle $D^{\prime}$ has a section and we are done. The same argument works if $\operatorname{dim} X^{\prime \prime}=2$ and $D^{\prime \prime 2} \neq 0$. In the remaining case that $\operatorname{dim} X^{\prime \prime}=2$ and $\left(D^{\prime \prime}\right)^{2}=0$, we need more arguments. Let $l^{\prime}$ be a smooth conic and assume that $l^{\prime}$ meets $C$. Let $l$ be its strict transform in $X$. Then $K_{X} \cdot l \geq-1$. Since $D \cdot l=0$ and $L \cdot l>0$, necessarily $K_{X} \cdot l=-1$ and $E \cdot l=1$. Thus $l$ meets $C$ transversely in one point. The same computations show that $C$ cannot meet a singular conic. Thus $C$ is a section of $X^{\prime} \rightarrow X^{\prime \prime}$ and $X \rightarrow X^{\prime \prime}$ is still a conic bundle. Then we conclude by Lemma 4.2 and Remark 4.3.

(d.2) Suppose $\phi^{\prime}$ is birational with exceptional divisor $E^{\prime}$.

If $C \subset E^{\prime}$, then, $C$ being rigid, $E^{\prime}$ must be ruled and $C$ is the exceptional section in $E^{\prime}$. Let $l^{\prime}$ be a ruling line and $l$ its strict transform in $X$. Then $K_{X} \cdot l=0$. Since $D \cdot l=0$, we obtain $L \cdot l=0$, which is absurd.

Things are more complicated when $E^{\prime} \cap C$ is a finite non-empty set. Suppose first that $E^{\prime}$ is not $\mathbb{P}_{2}$ with normal bundle $\mathcal{O}(-1)$. In this situation we find a rational curve $l^{\prime} \subset E^{\prime}$ meeting $C$ with $K_{X^{\prime}} \cdot l^{\prime}=-1$. Let $\hat{l}$ be the strict transform in $X$. Then

$$
\phi^{*}\left(l^{\prime}\right)=\hat{l}+a l
$$

with some positive integer $a$. Since $D^{\prime} \cdot l^{\prime}=D \cdot l=0$, it follows $D \cdot \hat{l}=0$. Now

$$
K_{X} \cdot \hat{l}=-1+a \geq 0 .
$$

Hence $D \cdot \hat{l}=\left(L+\mu K_{X}\right) \cdot l>0$, contradiction.

It remains to do the case $E^{\prime}=\mathbb{P}_{2}$ with normal bundle $\mathcal{O}(-1)$. Fixing a line $l^{\prime} \subset E^{\prime}$ which meets $C$, the same computations as above show that

$$
L \cdot \hat{l}=1, \mu=1, K_{X} \cdot \hat{l}=-1 \text { and } a=1 .
$$

Notice that $E^{\prime}$ can meet $C$ only in one point (transversely). In fact, otherwise we choose two points in $E^{\prime} \cap C$ and a line $l^{*}$ through these two points. Then the strict transform $\hat{l}^{*}$ satisfies $K_{X} \cdot \hat{l}^{*} \geq 0$, which is impossible, as already observed. Hence $\hat{E}^{\prime}$ is ruled over $\mathbb{P}_{1}$ with fibers $\hat{l}$. Since $\hat{E}^{\prime} \cdot \hat{l}=-1$, we can blow down $X$ along the projection $\hat{E}^{\prime} \rightarrow \mathbb{P}_{1}$ to obtain $\psi: X \rightarrow Y$, the blow-up of $Y$ along a smooth curve $C^{\prime} \simeq \mathbb{P}_{1}$. A priori it is not clear that $Y$ is projective. Let $L_{Y}=\left(\psi_{*}(L)\right)^{* *}$. Then

$$
L=\psi^{*}\left(L_{Y}\right)-\hat{E}^{\prime} .
$$

Denoting by $C_{0}$ the exceptional section of $\hat{E}^{\prime}$ and noticing that $N_{\hat{E}^{\prime}}^{*}=C_{0}+\hat{l}$, we obtain

$$
\left.L \mid \hat{E}^{\prime}=C_{0}+\left(L_{Y} \cdot C^{\prime}\right)+1\right) \hat{l} .
$$

Since $L \mid \hat{E}^{\prime}$ is ample, it follows that $L_{Y} \cdot C^{\prime}>0$ so that $L_{Y}$ is strictly nef on the Moishezon manifold $Y$. Then $Y$ has to be projective: otherwise by [Pe86] we find an irreducible curve $D$ and a positive closed current $T$ on $Y$ such that $[D+T]=0$. But $L_{Y} \cdot D>0$ and $L_{Y} \cdot T \geq 0$. Now, $Y$ being projective, we conclude by the first part of (b).

If finally $E^{\prime} \cap C=\emptyset$, then the strict transform of $E^{\prime}$ in $X$ is some $E_{j}, 2 \leq j \leq n$, hence defines an extremal contraction on $X$ with the same properties as $\phi$ and we can continue by induction. Since we assume $X$ uniruled, after finitely many steps we arrive at $\operatorname{dim} X^{[m]} \leq 2$ and argue as above. 


\section{Higher Dimensions}

In higher dimensions it is certainly very difficult to deal with Fano fibrations; however it is instructive to look at $\mathbb{P}_{k}$-bundles to get an idea on the higher dimensional case. Here we can calculate explicitly.

6.1. Theorem. Let $X$ be a $\mathbb{P}_{k}$-bundle over a smooth surface $S$. Suppose that $L$ is strictly nef on $X$. Then $K_{X}+t L$ is ample for $t>k+3$.

Proof. After possibly performing a finite étale cover, we may assume that $X$ is the projectivisation of a rank $(r+1)$-bundle $\mathcal{E}$ on $S$. If we allow $\mathcal{E}$ to be a $\mathbb{Q}$-bundle, we may assume that

$$
L=\mathcal{O}_{\mathbb{P}(\mathcal{E})}(k)
$$

with some positive number $k$. We also introduce $\zeta=\mathcal{O}_{\mathbb{P}(\mathcal{E})}(1)$. Notice that $\operatorname{det} \mathcal{E}$ is strictly nef and suppose that $K_{X}+t L$ is not ample. Then

$$
K_{X}^{j} \cdot L^{r+2-j}=0
$$

for all $j$ by (1.5). First recall the following

$$
\zeta^{r+1}-\pi^{*} c_{1}(\mathcal{E}) \zeta^{r}+\pi^{*} c_{2}(\mathcal{E}) \zeta^{r-1}=0
$$

and

$$
K_{X}=-(r+1) \zeta+\pi^{*}\left(\operatorname{det} \mathcal{E}+K_{S}\right) .
$$

The equation $L^{r+2}=0$ immediately leads to

$$
\zeta^{r+2}=c_{1}(\mathcal{E})^{2}-c_{2}(\mathcal{E})=0 .
$$

Secondly, combining with $\zeta^{r+2}=0$, the equation $L^{r+1} \cdot K_{X}=0$ leads to

$$
\zeta^{r+1} \cdot \pi^{*}\left(c_{1}(\mathcal{E})+K_{S}\right)=c_{1}(\mathcal{E}) \cdot\left(c_{1}(\mathcal{E})+K_{S}\right)=0 .
$$

Moreover, the equation $L^{r} \cdot K_{X}^{2}=0$ leads to

$$
\zeta^{r} \cdot \pi^{*}\left(c_{1}(\mathcal{E})+K_{S}\right)^{2}=\left(c_{1}(\mathcal{E})+K_{S}\right)^{2}=0 .
$$

By $(6),(7)$, we have $K_{S} \cdot\left(c_{1}(\mathcal{E})+K_{S}\right)=0$ and hence $K_{S}^{2}=c_{1}(\mathcal{E})^{2}$. Since $\operatorname{det} \mathcal{E}$ is strictly nef, equation $(6)$ yields that $K_{S}^{2}=c_{1}(\mathcal{E})^{2} \geq 0$ and $c_{1}(\mathcal{E}) \cdot K_{S} \leq 0$.

First suppose that $\kappa(S) \geq 0$. Then $K_{S} \cdot \operatorname{det} \mathcal{E}=0$ and $K_{S}^{2}=0$ for $\operatorname{det} \mathcal{E}$ being strictly nef. Hence $K_{S} \equiv 0$. Then by $(1.5) \operatorname{det} \mathcal{E}$ is ample, contradicting $c_{1}(\mathcal{E})^{2}=$ $K_{S}^{2}$.

It remains to consider $\kappa(S)=-\infty$. Since $K_{S}^{2} \geq 0, S$ is either rational or a minimal ruled surface over an elliptic curve. In the latter case, $K_{S}^{2}=0$, hence $c_{1}(\mathcal{E})^{2}=0$. On the other hand, any strictly nef divisor on a ruled surface over an elliptic curve is ample (use [Ha77,V.2]), a contradiction.

In case of a rational surface $S$, choose a positive integer $m$ such that $m \operatorname{det} \mathcal{E}$ is Cartier. Then Riemann-Roch and $\left(K_{S}+\operatorname{det} \mathcal{E}\right)^{2}=0$ show that $h^{0}\left(m\left(K_{S}+\operatorname{det} \mathcal{E}\right)\right)>$ 0 . This contradicts via $\left(K_{S}+\operatorname{det} \mathcal{E}\right) \cdot \operatorname{det} \mathcal{E}=0$ the strict nefness of $\operatorname{det} \mathcal{E}$.

\section{REFERENCES}

[BDPP04] Boucksom, S.; Demailly, J.P.; Paun, M.; Peternell, Th.: The pseudo-effective cone of a compact Kähler manifold and varieties of negative Kodaira dimension. math.AG/0405285

[Ha04] Hacon, C.: A derived category approach to generic vanishing. J. Reine Angew. Math. 575, 173-187 (2004). 
[Ha70] Hartshorne, R.:Ample subvarieties of algebraic varieties. Lecture Notes in Mathematics, 156, Springer Verlag, (1970).

[Ha77] Hartshorne, R.:Algebraic geometry. Graduate Texts in Mathematics, 52, SpringerVerlag, (1977).

[Ko95] Kollár, J.: Shafarevich maps and automorphic forms. Princeton University Press, (1995).

[Ko92] Kollár, J.: Flips and abundance for algebraic threefolds. Astérisque 211 (1992)

[KM98] Kollár, J.; Mori, S.: Birational geometry of algebraic varieties. Cambridge Tracts in Mathematics, 134. Cambridge University Press, Cambridge, (1998)

[KMM87] Kawamata, Y.; Matsuki, K.; Matsuda, K. Introduction to the minimal model program. Adv. Stud. Pure Math. 10 (1987), 283-360

[Mo82] Mori,S.: Threefolds whose anticanonical bundles are not numerically effective. Ann Math. 116, 133-176 (1982)

[My83] Miyanishi, M.: Algebraic threefolds. Adv. Stud. Pure Math. 1, 69-99 (1983)

[Mu81] Mukai, S.: Duality between $D(X)$ and $D(\hat{X})$ with application to Picard sheaves. Nagoya Math. J. 81, 153-175 (1981)

[Pe86] Peternell, T.: Algebraicity criteria for compact complex manifolds. Math. Ann. 275, 653-672 (1986)

[Se95] Serrano, F.: Strictly nef divisors and Fano threefolds. J. Reine Angew. Math. 464, 187-206 (1995).

Frédéric Campana, Département de Mathématiques, Université de Nancy, F-54506 Vandoeuvre-les-Nancy, France,

frederic.campana@iecn.u-nancy.fr

Jungkai Alfred Chen, Department of Mathematics, National Taiwan University, Taipei 106, Taiwan,

jkchen@math.ntu.edu.tw

Thomas Peternell, Mathematisches Institut, Universität Bayreuth, D-95440 Bayreuth, thomas.peternell@uni-bayreuth.de 